Áreas Naturales Protegidas Scripta, 2018. Vol. 4 (2): 25-47. https://doi.org/10.18242/anpscripta.2018.04.04.01.0002

Habitat, Corridors and Scenarios Modeling for Ecosystem Service Assessment in Protected Natural Areas of the Sierra Madre de Chiapas

\title{
Modelado de Hábitat, Corredores y Escenarios para la Valoración de Servicios Ecosistémicos en Áreas Naturales Protegidas de la Sierra Madre de Chiapas
}

Sandra Lizbeth Medina-Fernández ${ }^{1}$,

Catalina Ordoñez-Ramos ${ }^{1}$, Juan Manuel Núñez ${ }^{2 *}$

\section{Resumen}

Preservar los ecosistemas a través de las Áreas Naturales Protegidas proporciona servicios imprescindibles para el bienestar humano. Por lo tanto, adoptar el marco de la valoración de los servicios ecosistémicos como herramienta de ayuda para la toma de decisiones, permite mejorar la protección de la diversidad biológica y la gestión de las Áreas Naturales Protegidas. El presente trabajo contribuye al mapeo y valoración de servicios ecosistémicos dentro y fuera de las Áreas Naturales Protegidas en la Sierra Madre de Chiapas en México. A través del uso de herra de modelado espacial para la simulación de escenarios proyectados de uso de suelo y vegetación, se evalúa la vulnerabilidad de la pérdida de hábitat de tapir (Tapirus bairdii) a partir de la integración de un modelo de calidad de hábitat y el diseño de corredores para el modelado de parches de hábitat. Mediante los escenarios obtenidos, se observa que el cambio a futuro no será muy intenso, por lo que se asegura la permanencia del corredor propuesto dentro de los años evaluados.

Palabras clave: Modelado Espacial. Servicios Ecosistémicos. Áreas Naturales Protegidas. Calidad de Hábitat. Conectividad.

\footnotetext{
${ }^{1}$ Universidad Nacional Autónoma de México -Facultad de Ingeniería - División de Ingeniería Civil y Geomática.

${ }^{2}$ Centro de Investigación en Ciencias de Información Geoespacial (CentroGeo)

*jnunez@centrogeo.edu.mx
} 


\section{Abstract}

Preserving ecosystems through protected natural areas provides essential services for human wellbeing. Thus, adopting the ecosystem service valuation framework as a tool to aid decision-making improves biodiversity protection and management of the protected natural areas. This study contributed to mapping and valuating ecosystem services outside and inside of the Protected Natural Areas in the Sierra Madre de Chiapas, Mexico. Through spatial simulation of projected scenarios of land use and land cover, the vulnerability of the tapir (Tapirus bairdii) habitat loss was assessed from integrating a habitat quality model to designing corridors for modeling habitat patches. Thus, the scenarios showed that change in the future would not be very noticeable in each land use and vegetation, ensuring the persistence of the corridor within the evaluated years.

Key words: Spatial Modeling. Ecosystem Services. Protected Areas. Habitat Quality. Connectivity.

\section{Introducción}

Atender los problemas en relación con la conservación de los ecosistemas naturales, la diversidad biológica que contienen y los servicios ecosistémicos que nos brindan, es un asunto de seguridad nacional que debe tener un lugar preponderante en el plan de desarrollo del país (Sarukhán et al., 2012). En México, las Áreas Naturales Protegidas (ANP) desempeñan un papel fundamental en la provisión de servicios ecosistémicos necesarios para la transferencia de beneficios económicos, sociales, culturales y ambientales que trascienden a su delimitación geográfica e impactan en el bienestar de todos sus pobladores (CONANP, 2015).

La valoración de los servicios de los ecosistemas es un enfoque adecuado para evaluar la efectividad de la conservación de las ANP, incluyendo la consideración de las actividades humanas existentes en las tierras circundantes (Martín-López et al., 2011). Por otra parte, el uso de Sistemas de Información Geográfica (SIG) se ha convertido en una herramienta de ayuda para analizar patrones espacio-temporales de los servicios que ofrecen los ecosistemas y estimar el impacto potencial de los cambios proyectados sobre el uso del suelo y la vegetación (Nemec et al., 2013). El manejo de información geoespacial permite mapear y evaluar de forma cuantificada los beneficios 
proporcionados por los diferentes ambientes y su biodiversidad para gestionar su conservación, obtener un mejor aprovechamiento de los recursos o evaluar el daño ocasionado por acciones antropogénicas.

En este trabajo, utilizamos el enfoque de servicios ecosistémicos para evaluar la gestión del uso del suelo dentro y fuera de las ANP en términos de conectividad, basados en el uso de herramientas de SIG sobre la calidad de hábitat, el diseño de corredores y la generación de escenarios de uso del suelo para conocer su estado actual y futuro. Específicamente, (1) construimos un modelo de calidad de hábitat con base a los usos de suelo y vegetación para el tapir (Tapirus bairdii) en el complejo de ANP de la Sierra Madre de Chiapas en México; (2) diseñamos un corredor entre ANP para el traslado del tapir y la protección del paisaje intermedio; (3) generamos un escenario prospectivo al 2039, de los usos de suelo y vegetación para analizar las pérdidas y ganancias de coberturas dentro del corredor propuesto y verificar su permanencia en el futuro cercano; y (4) evaluamos el papel de las ANP y sus alrededores en la conservación de los ecosistemas y el servicio de provisión de hábitat que otorgan.

\section{Materiales y Métodos}

Área de estudio

El área de estudio es la Sierra Madre de Chiapas (SMC), una cadena montañosa orientada de noroeste a sureste que se extiende paralela a la costa del pacífico en el sur de México. Está conformada por 36 municipios del estado de Chiapas, con una superficie de 2,608,950 ha. Dentro de la parte alta de la SMC se encuentran cuatro ANP de carácter federal: la Zona de Protección Forestal La Frailescana; además de las Reservas de la Biosfera La Sepultura, El Triunfo, y Volcán Tacaná. En conjunto estas ANP tiene una superficie decretada de más de 470,000 ha (Fig. 1).

Se trata de una región de gran importancia para la conservación de la biodiversidad, acogiendo más de 2,000 especies de plantas y por lo menos 600 especies de vertebrados terrestres (CortinaVillar et al., 2012). En esta área coexisten al menos 13 especies de mamíferos de tamaño medianogrande, que incluyen cuatro especies de ungulados (Tapirus bairdii, Mazama temama, Odocoileus virginianus y Pecari tajacu) y felinos como jaguares (Panthera onca) y puma (Puma concolor) (VazquézBautista, 2002). 


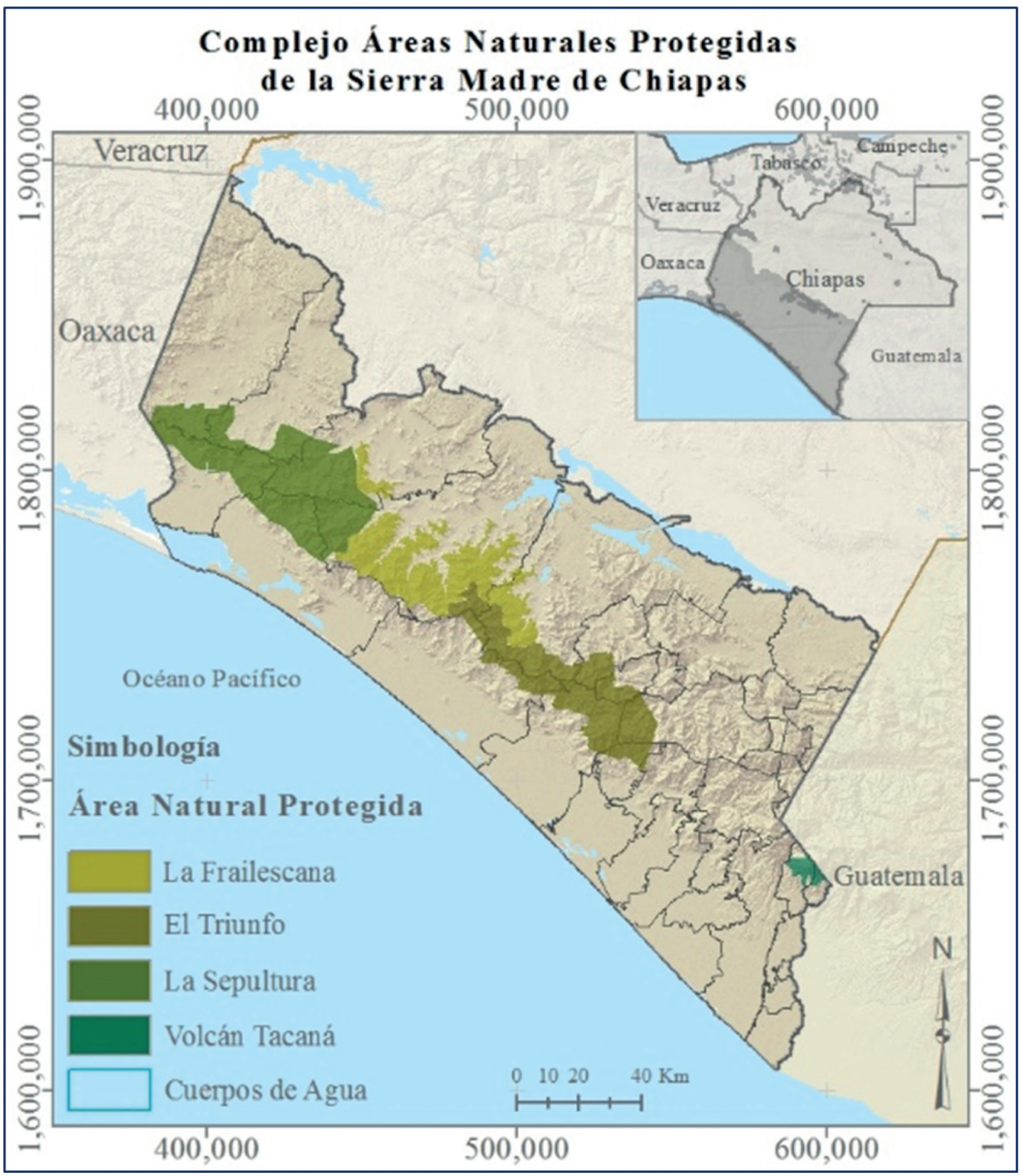

Figura 1. Ubicación geográfica del área de estudio. Elaboración propia.

\section{Metodología}

Al modelar procesos ecosistémicos, los Sistemas de Información Geográfica (SIG) son útiles ya que tienen la capacidad de incorporar las complejidades de las dimensiones espaciales involucradas, lo que nos permite visualizar el patrón y la distribución de los elementos del paisaje 
ecológicamente importantes y superponerlos con otros temas relevantes (Grêt-Regamey et al., 2008; Troy y Wilson, 2006). Con el crecimiento reciente en el campo de los servicios ecosistémicos, ha surgido una variedad de herramientas SIG para respaldar una evaluación más sistemática de los servicios ecosistémicos. Mientras que algunas de estas herramientas permiten integrar dichos modelos de procesos de ecosistemas en una plataforma SIG (Boll et al., 2004; Eade y Moran, 1996), algunas otras han ido un paso más allá y permiten vincular los procesos de cuantificación del servicio ecosistémico con diferentes métodos de valoración dentro de un ambiente SIG (Laterra et al., 2016; Sharp et al., 2016; Villa et al., 2007). Sin embargo, ninguna de estas herramientas son lo suficientemente amplias para integrar en una sola plataforma varios modelos de procesos de ecosistemas que simulen la provisión de servicios ecosistémicos simultáneamente con los diversos procedimientos de valoración.

Bajo un enfoque de servicios ecosistémicos, en este trabajo se integran diversos modelos basados en procesos que utilizan SIG para evaluar la efectividad de la conservación de las ANP, incluyendo la consideración de las actividades humanas existentes en las tierras circundantes. El marco, que se basa en el uso de herramientas de modelado espacial, está integrado por tres pasos: (i) el modelado de calidad de hábitat de tapir (Tapirus bairdii) mediante el uso de modelos determinísticos implementados en la plataforma InVEST; (ii) el diseño de corredores para el modelado de parches de hábitat a partir de la herramienta CorridorDesign; y (iii) la simulación de escenarios proyectados de uso de suelo y vegetación mediante el uso de redes neuronales implementadas en la herramienta Land Change Modeler para evaluar la vulnerabilidad de la pérdida de hábitat del tapir (Tapirus bairdii) en el futuro cercano. Debido a que estas herramientas están plenamente orientadas al análisis de los ecosistemas, todas se integraron dentro de un marco metodológico, tal como se muestra en la Fig. 2.

\section{Modelo de Calidad de Hábitat}

Integred Valuation of Enviroment Services and Tradeoffs (InVEST) es una aplicación de licencia libre que utiliza el ambiente SIG como interfaz para integrar los datos de entrada y visualizar los resultados. Se trata de una herramienta que combina información de la cobertura de uso de suelo y vegetación (USV) y las amenazas a la biodiversidad para producir mapas de calidad de hábitat. Esta acción genera dos clases de información clave para realizar una evaluación inicial de las 
necesidades de conservación: la extensión y degradación relativas a diferentes tipos de hábitat en una región y los cambios a través del tiempo. Dentro de este modelo, la calidad de hábitat se refiere a la habilidad de los ecosistemas para proveer condiciones apropiadas para la persistencia individual o de una población, y se considera una variable continua, con base en los recursos disponibles para la supervivencia, reproducción y persistencia de la población respectivamente (Sharp et al., 2016).

\section{Recopilación de información}

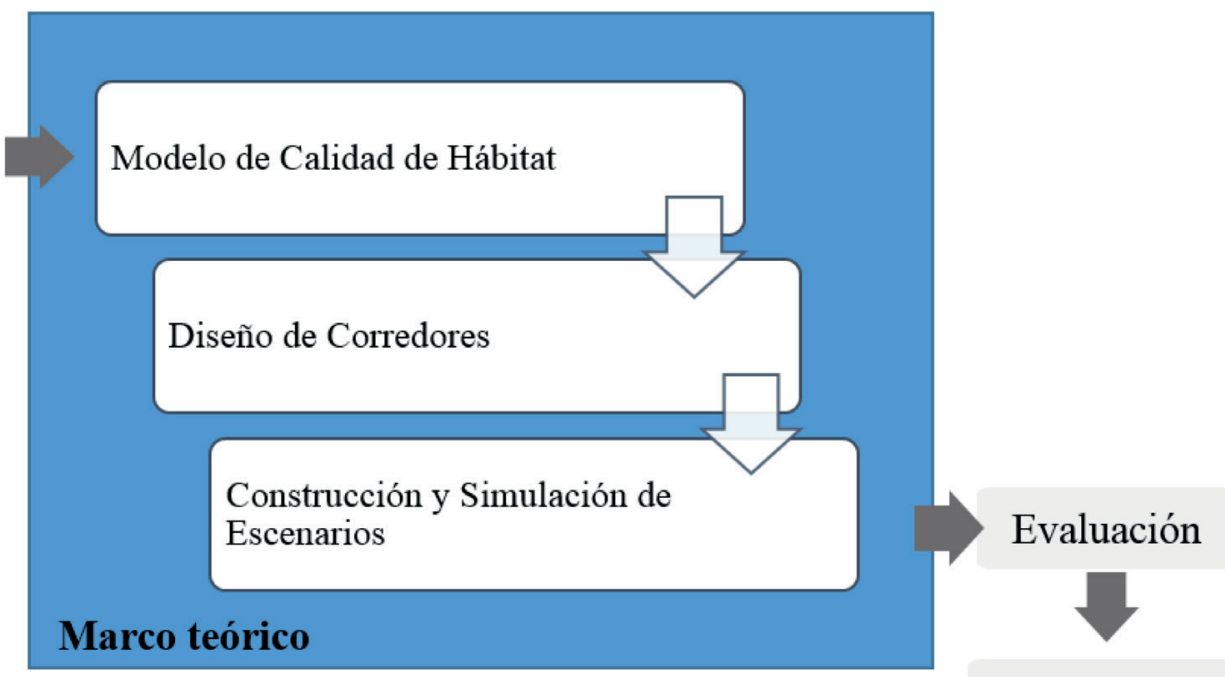

Conclusiones

Figura 2. Metodología para el desarrollo del modelo de calidad de hábitat, corredores y escenarios para la evaluación y mapeo de servicios ecosistémicos. Elaboración propia.

El modelo para su implementación requiere de los siguientes insumos:

-Cobertura de USV para un tiempo actual. Para este trabajo se empleó la serie V de USV para el año 2011, elaborada por el Instituto Nacional de Estadística y Geografía (INEGI, 2013a), la cual fue categorizada en veintinueve clases (Selva Perennifolia Primaria Arbórea, Selva Perennifolia Secundaria Arbustiva, Selva Perennifolia Secundaria Arbórea, Bosque Mesófilo de Montaña Primario Arbóreo, Bosque Mesófilo de Montaña Secundario Arbustivo, Bosque Mesófilo de Montaña Secundario Arbóreo, Selva Caducifolia Primaria Arbórea, Selva Caducifolia Secundaria Arbustiva, Selva Caducifolia Secundaria Arbórea, Selva Espinosa Secundaria Arbustiva, Selva Subcaducifolia Secundaria Arbustiva, Selva Subcaducifolia Secundaria Arbórea, Bosque de Encino Primario Arbóreo, Bosque de Encino Secundario Arbóreo, Otros 
Tipos de Vegetación, Pastizal Primario Herbáceo, Bosque de Coníferas Primario Arbóreo, Bosque de Coníferas Secundario Arbustivo, Bosque de Coníferas Secundario Arbóreo, Agricultura de Riego Anual, Agricultura de Riego Semipermanente, Agricultura de Temporal Anual, Agricultura de Temporal Permanente, Agricultura de Temporal Semipermanente, Cuerpo de Agua, Desprovisto de vegetación, Pastizal Pecuario Cultivado Permanente, País Extranjero y Zona urbana).

- Información sobre los requerimientos de hábitat del tapir (Tapirus bairdii) para la Sierra Madre de Chiapas, en donde se reporta que a partir de un conteo de huellas y heces se observó que los tapires prefieren áreas densas de selva mediana subperennifolia y bosques mesófilo de montaña con arroyos y estanques permanentes. Sin embargo, el tapir generalmente evita los hábitats secos, abiertos y perturbados como el bosque de pino y pastizales. Además el tapir ocupa una amplia variedad de Selvas, Bosques y Humedales cuya altitud varía entre 0 a 2,000 msnm. (March y Naranjo, 2005; Naranjo y Cruz, 1998).

- Identificación y sensibilidad de amenazas, referida como el establecimiento de amenazas y su afectación hacia cada tipo de USV. Asignando así, valores de '1' al Uso de Suelo y Vegetación que son hábitat para el Tapir y en el caso contrario un '0'; después, se determinó una calificación (el valor mayor indica una alta preferencia por ese tipo de hábitat) a cada categoría, para después fijar un rango del 1.1 al 1.7, este rango permitió identificar qué tipos de USV pueden ser más afectados que otros por las amenazas (donde el 1.7 es el más bajo, es decir, el menos afectado). Posteriormente, se le dio una puntuación de sensibilidad que cada amenaza afecta a cada USV, es decir que a mayor sensibilidad ante una determinada amenaza en un tipo de hábitat, mayor será la degradación de este tipo de hábitat a causa de esa amenaza, donde '1' representa una alta sensibilidad a una amenaza y ' 0 ' no representa ninguna sensibilidad. Para ese trabajo fueron designadas como amenazas diferenciadas las localidades urbanas y rurales, las vialidades con y sin revestimiento, las zonas pecuarias y agrícolas y la elevación mayor a 2,000 msnm.

De esta forma, se obtienen las zonas con calidad de hábitat para el tapir (Tapirus bairdii) en un gradiente de degradación. Las cuales, son utilizadas como entrada para el diseño de corredores que maximiza la conectividad entre las ANP de El Triunfo y La Sepultura a través de La Frailescana. 


\section{Diseño de Corredores}

Para el diseño de corredores se utilizó la herramienta CorridorDesign, con el objetivo de diseñar corredores biológicos cuyo fin es proporcionar conectividad entre paisajes, ecosistemas y hábitat, naturales o modificados, para asegurar el mantenimiento de la biodiversidad y los procesos ecológicos y evolutivos (Jenness et al., 2011).

Para el desarrollo del modelo fue necesaria la normalización del mapa de calidad de hábitat generado previamente en InVEST y la recopilación de información complementaria acerca de la especie involucrada que define el tamaño del umbral para el hábitat adecuado, el tamaño mínimo de cría y de población. Una vez normalizados los valores de degradación se construyó el mapa de parches, el cual, evalúa los valores de los pixeles en un radio definido. En cuanto al tamaño del umbral para el hábitat adecuado se seleccionó el valor de cincuenta hectáreas ya que es la unidad mínima cartografiable de las comunidades vegetales en el mapa de USV (INEGI, 2013a). Para los tamaños mínimos por cría y por población se encontraron diversas variaciones, debido principalmente a la disponibilidad y condiciones de hábitat en el que se encuentran. En la literatura especializada se ha reportado la abundancia de tapir en un área de muestreo de aproximadamente 0.09 individuos por $\mathrm{km}^{2}$ (Mendoza-Ramírez y Borges, 2011), mientras que para los ámbitos hogareños se reporta entre $0.62 \mathrm{~km}^{2}$ y $2.32 \mathrm{~km}^{2}$ (Foerster, 1998), siendo mayores para los tapires machos que para las hembras $\left(1.60 \mathrm{~km}^{2}\right.$ y $1.02 \mathrm{~km}^{2}$ respectivamente). Con base en lo anterior, se consideró un tamaño mínimo de cría de 11 ha y 62 ha por población de acuerdo a la literatura referida.

\section{Generador de Escenarios}

La herramienta Land Change Modeler (LCM) para la sostenibilidad ecológica es un modelo orientado principalmente a los problemas del cambio de uso del suelo y de las necesidades de análisis de conservación de la biodiversidad (Eastman, 2006). El modelo permite elaborar una proyección espacialmente explícita hacia el futuro como posibles hechos, es decir, futuros inciertos, pero útiles principalmente para la toma de decisiones y su evaluación (Sharp et al., 2016).

Para ello es necesario contar con mapas de uso de suelo y vegetación en dos tiempos, pasada y actual. Por lo que, para este trabajo se consideraron como insumos las series II y V de USV 
para los años 1993 y 2011 respectivamente (INEGI, 1997; INEGI, 2013a). A partir de las 29 clases descritas anteriormente, se realizó una homologación a siete clases de cobertura (Agrícola, Agua, Bosques y selvas, Asentamientos humanos, Otra vegetación, Otros usos y Pecuario) para cada uno de los mapas de USV de 1993 (Fig. 3) y 2011 (Fig. 4).

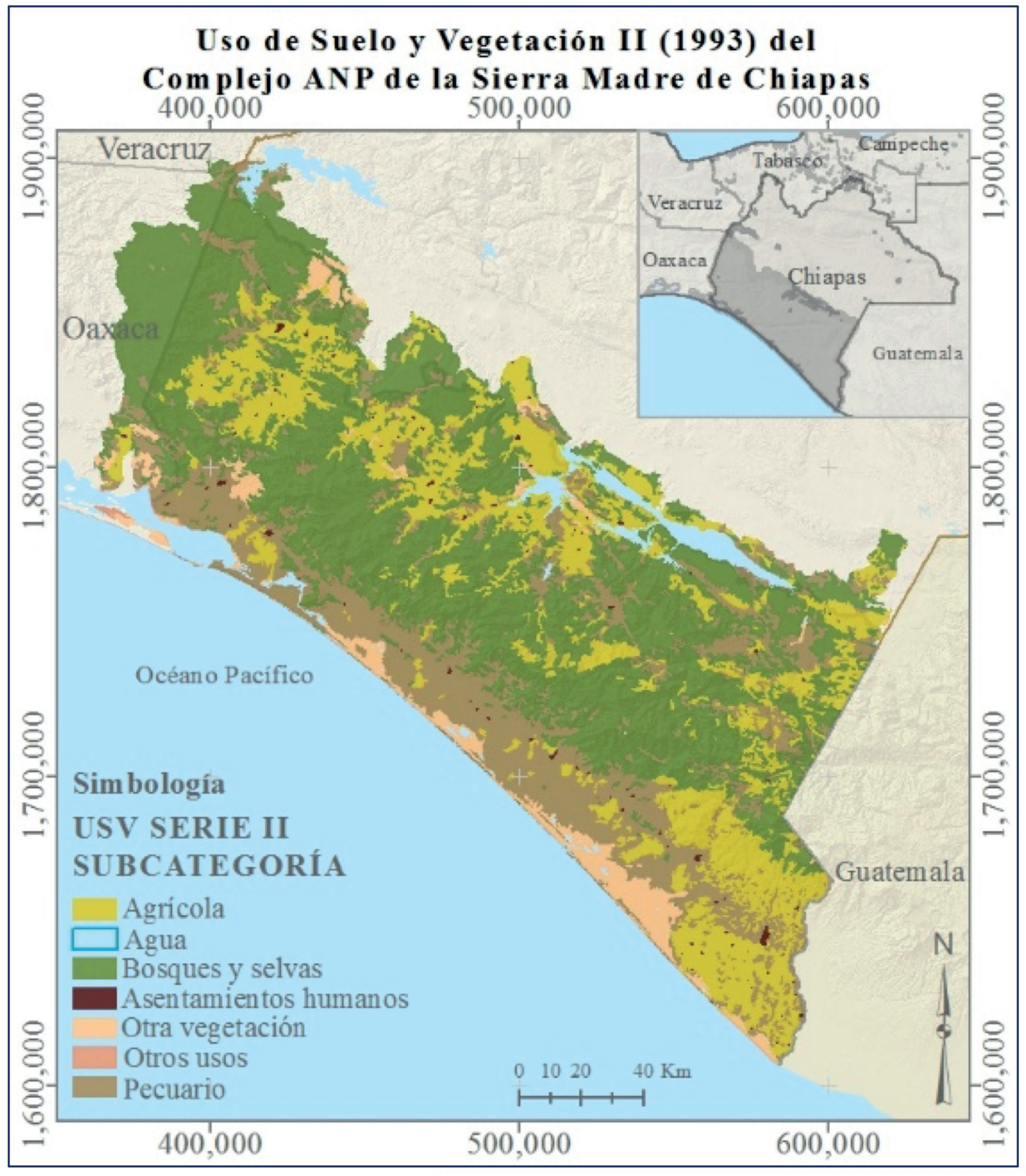

Figura 3. Mapa de USV para el año 1993 del Complejo de ANP de la Sierra Madre de Chiapas. Elaboración propia. 


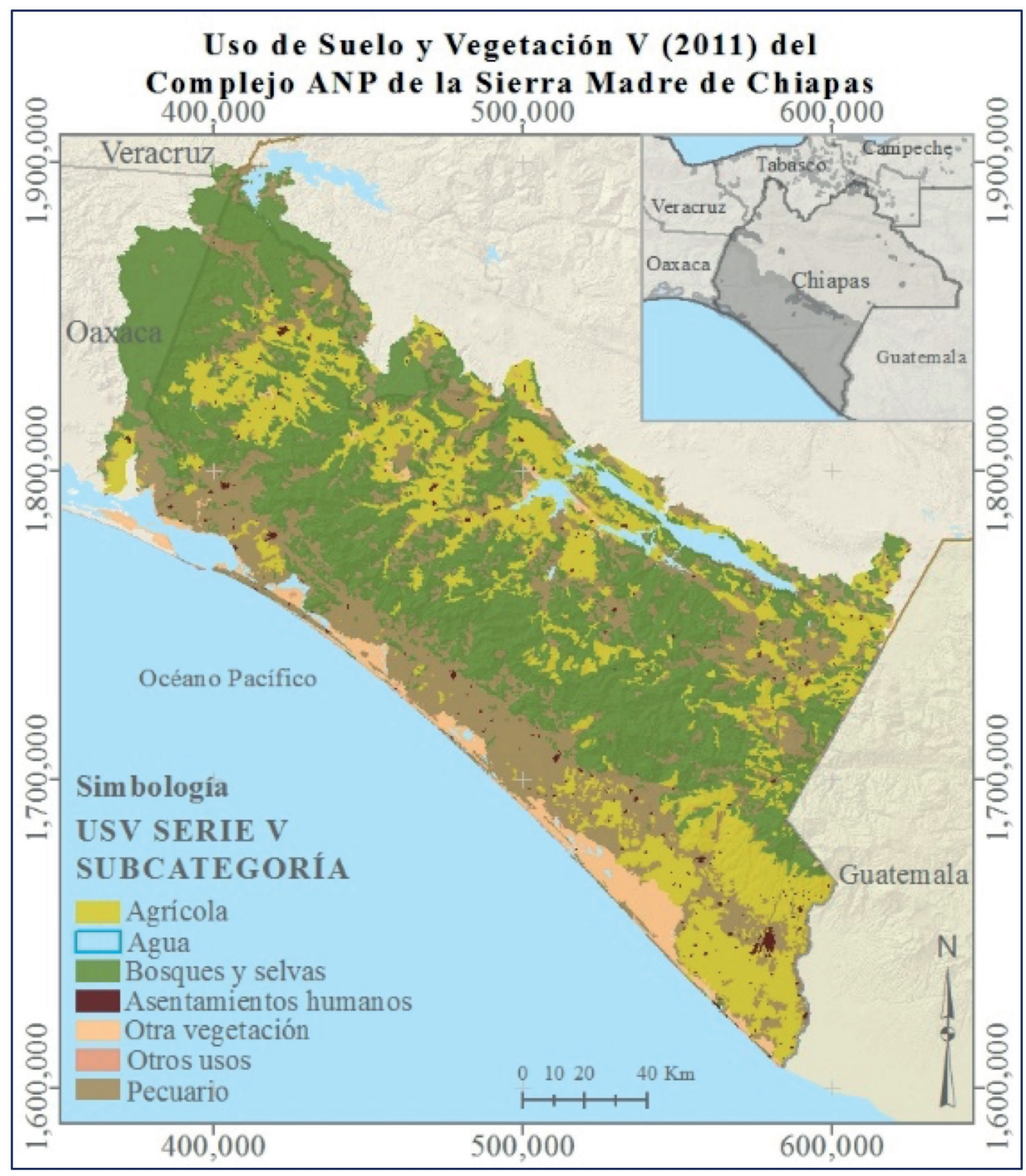

Figura 4. Mapa de USV para el año 2011 del Complejo de ANP de la Sierra Madre de Chiapas. Elaboración propia.

A continuación, se realizó un análisis de cambio para establecer las diferencias registradas entre las distintas coberturas de USV, como se muestra en la Fig. 5. 


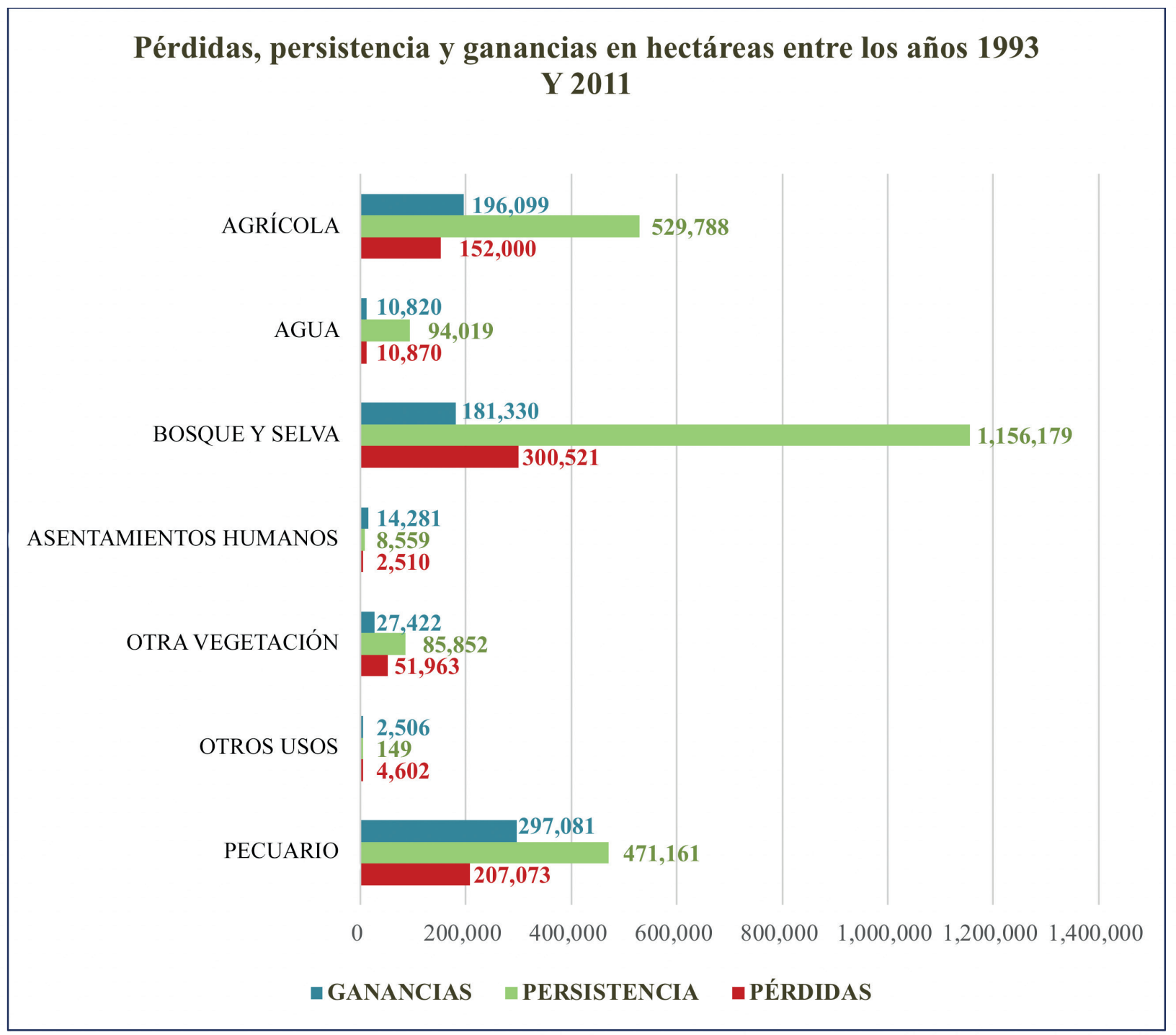

Figura 5. Pérdidas, persistencia y ganancias en el área de estudio. Elaboración propia.

El análisis de cambio permite representar las pérdidas, ganancias y persistencia de las cubiertas ingresadas, pudiéndose observar mayor superficie de persistencia en Bosques y Selvas; sin embargo, son también las categorías donde se ha perdido la mayor cantidad de hectáreas, mismas que se ven reflejadas principalmente en las ganancias de las categorías Agrícola y Pecuario. Una vez realizado el análisis espacial de cambios de USV, se procedió a identificar una serie de variables para ayudar a explicar las transiciones observadas mediante la aplicación de redes neuronales multicapa, empleadas para modelar de forma generativa la formación de patrones espacio-temporales de cambio en un escenario futuro (Mas et al., 2014). 
Las variables explicativas seleccionadas se describen a continuación:

-La densidad de población, la cual representa la tasa de crecimiento a partir del Censo de Población y Vivienda 2010, con respecto del Censo General de Población y Vivienda 1990 (INEGI, 1990; INEGI, 2010).

-Redes viales, a partir de la información disponible de la Secretaría de Comunicaciones y Trasporte (SCT) con datos de 2004 y 2008. A partir de estos datos, se obtuvieron dos variables: el tipo de vialidad categorizado como vialidades primarias y secundarias, además de la distancia a carreteras que funciona como variable de afectación en función a la cercanía.

-La pendiente del terreno, obtenida a partir de un Modelo Digital de Elevación, descargado de INEGI (2013b), cuya función es ayudar en la predicción del crecimiento de los factores antropogénicos.

Una vez establecidas las amenazas y determinada la fecha a predecir en un futuro cercano (2039), se generó el escenario prospectivo a partir del análisis espacial de cambios de cobertura y uso del suelo para el área de estudio, mediante un modelo de redes neuronales multicapa que incorpora una serie de variables que explican las transiciones observadas.

\section{Resultados y discusión}

De acuerdo a la evaluación en la identificación y sensibilidad de amenazas propuestas en el modelo InVEST, se obtuvieron zonas con calidad de hábitat para el tapir (Tapirus bairdii) en un gradiente de degradación (Fig. 6). En donde las áreas ya degradadas presentan un tono en negro, y en verde las áreas sin degradación. De acuerdo a nuestros resultados, se puede observar que las zonas con mayor calidad de hábitat se encuentran ubicadas en la parte central de la zona de estudio y en el límite con el estado de Oaxaca, mientras que las zonas de menor calidad de hábitat se encuentran sobre el límite con Guatemala. 


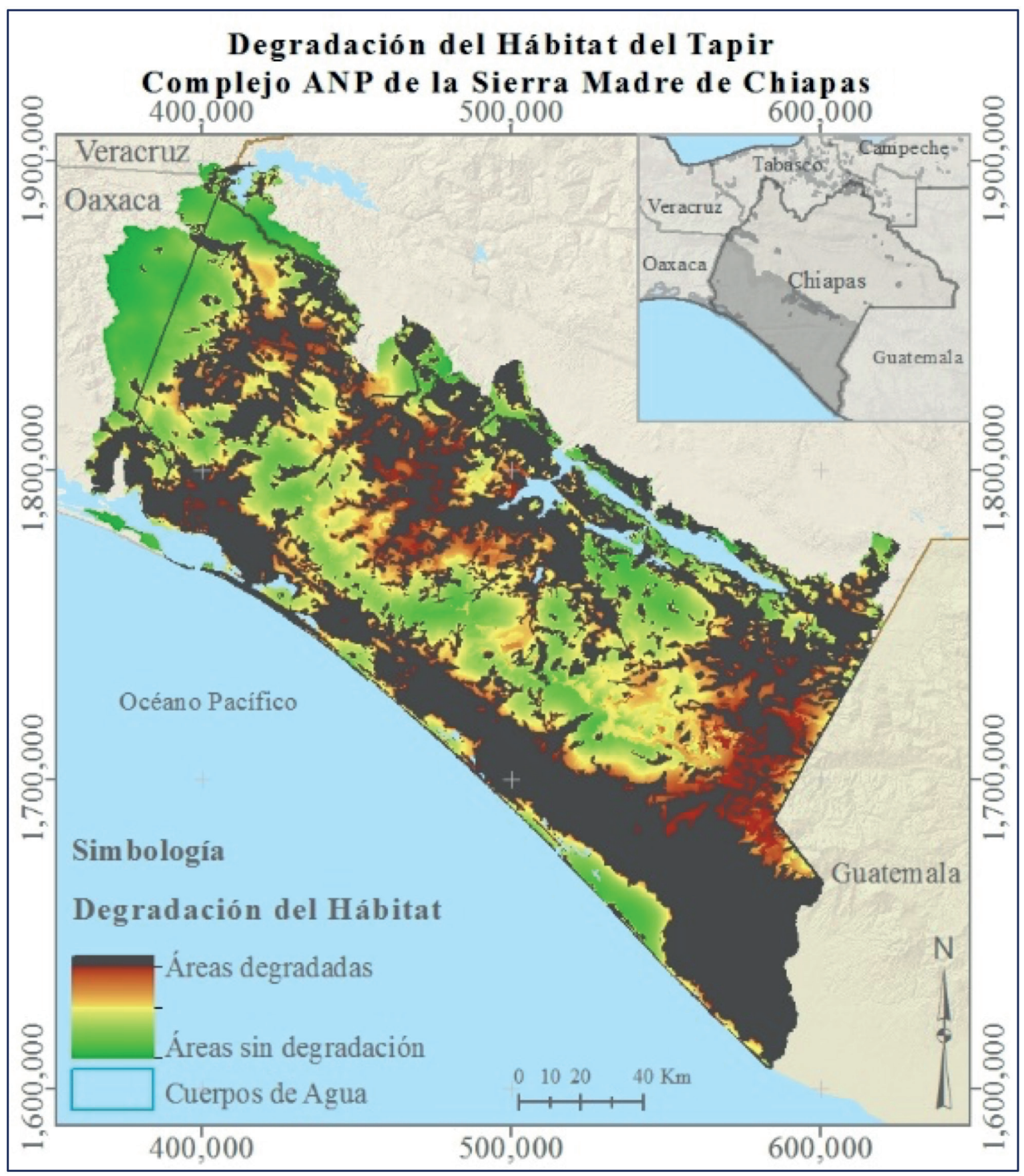

Figura 6. Degradación del hábitat del Tapir. Elaboración propia.

Este resultado, fue utilizado para diseñar corredores que maximizan la conectividad entre la distribución de los parches más significativos por tamaño de área de población. A partir de los datos ingresados y los parche generados, se diseñaron diferentes corredores procurando la conectividad entre las zonas núcleo de las ANP de La Sepultura y El Triunfo. A partir de estos resultados, se evaluaron once corredores con las siguientes características: 
Tabla I. Evaluación de corredores conforme a la Calidad de Hábitat y degradación. Elaboración propia.

\begin{tabular}{|c|c|c|c|c|c|}
\hline \multicolumn{6}{|c|}{ EVALUACIÓN DE CORREDORES } \\
\hline CORREDOR & $\begin{array}{c}\text { ÁREA } \\
\text { TOTAL (ha) }\end{array}$ & $\begin{array}{l}\text { CALIDAD DE } \\
\text { HÁBITAT (ha) }\end{array}$ & $\begin{array}{l}\text { CALIDAD DE } \\
\text { HÁBITAT (\%) }\end{array}$ & DEGRADACIÓN (ha) & DEGRADACIÓN (\%) \\
\hline$A=0.1$ & $45,798.84$ & $44,059.91$ & 96.2 & $1,738.93$ & 3.8 \\
\hline$B=1.0$ & $72,067.15$ & $69,892.36$ & 96.98 & $2,174.79$ & 3.02 \\
\hline$C=2.0$ & $97,761.91$ & $94,445.60$ & 96.61 & $3,316.31$ & 3.39 \\
\hline $\mathrm{D}=3.0$ & $123,463.61$ & $118,126.01$ & 95.68 & $5,337.60$ & 4.32 \\
\hline $\mathrm{E}=4.0$ & $147,672.00$ & $140,252.20$ & 94.98 & $7,419.80$ & 5.02 \\
\hline $\mathrm{F}=5.0$ & $170,482.51$ & $160,847.90$ & 94.35 & $9,634.61$ & 5.65 \\
\hline $\mathrm{G}=6.0$ & $193,035.67$ & $180,745.24$ & 93.63 & $12,290.43$ & 6.37 \\
\hline $\mathrm{H}=7.0$ & $214,939.86$ & $199,680.07$ & 92.9 & $15,259.79$ & 7.1 \\
\hline $\mathrm{I}=8.0$ & $236,393.63$ & $217,540.26$ & 92.02 & $18,853.37$ & 7.98 \\
\hline $\mathrm{J}=9.0$ & $256,221.30$ & $233,738.68$ & 91.23 & $22,482.62$ & 8.77 \\
\hline$K=10$ & $275,071.00$ & $248,973.29$ & 90.51 & $26,097.70$ & 9.49 \\
\hline
\end{tabular}

Finalmente, se seleccionó un único corredor a partir del criterio de mayor área disponible con menor degradación, seleccionando el corredor G. Siendo que resultó ideal dada su gran extensión territorial de un área total de 193,035.67 ha, de las cuales, el 7.2\% de superficie se encuentra fuera de las ANP lo que ofrece mayor seguridad en cuestión de permanencia para el corredor, ya que forma un camino amplio para el traslado del Tapir con los requerimientos suficientes para su conservación entre las zonas núcleo de La Sepultura y El Triunfo a través de La Frailescana, el cual se muestra a continuación en la Fig. 7. 


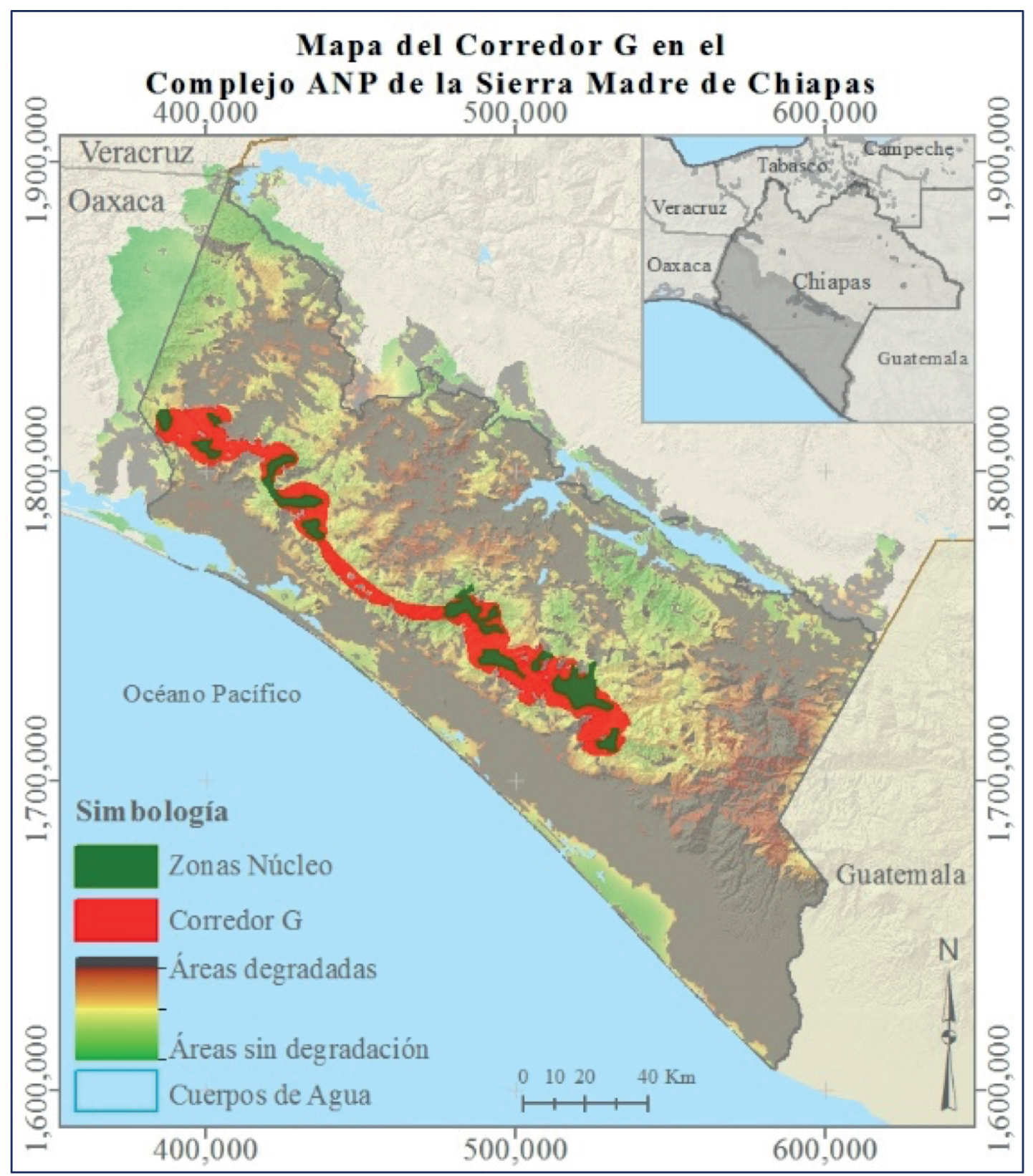

Figura 7. Resultado del corredor seleccionado a partir de Calidad de Hábitat y las zonas núcleo de las ANP La Sepultura y El Triunfo. Elaboración propia.

Como se observa en el mapa anterior y en la Fig. 8, la superficie ideal para el Tapir conforme a la Calidad de Hábitat y degradación no se encuentra completamente dentro de las Áreas Naturales Protegidas, siendo que La Frailescana no cubre en su totalidad el corredor obtenido, además de que se presentan amenazas muy significativas como son redes viales y densidad de población dentro de este.

Es por ello, la importancia de la generación de escenarios que representen los cambios a futuro de los USV para analizar la posibilidad de permanencia del corredor en las zonas que no entran en 


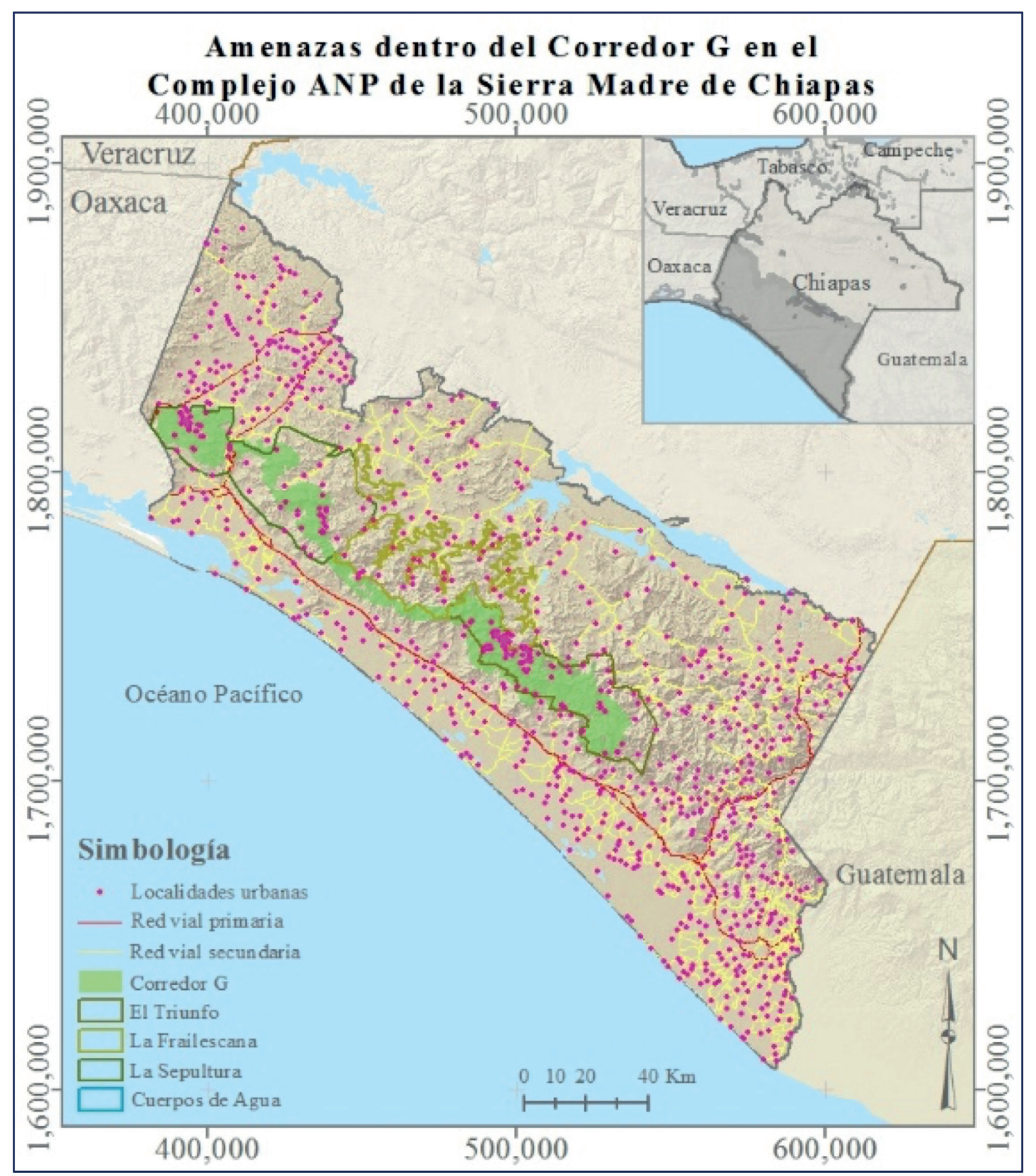

Figura 8. Calidad de hábitat y amenazas dentro del Corredor diseñado para el Tapir en las ANP La Sepultura, La Frailescana y El Triunfo. Elaboración propia.

las Áreas Naturales Protegidas. El mapa obtenido para el año 2039, se muestra en la Fig. 9.

El resultado de la predicción presenta cambios dentro y fuera de las ANP, los cuales pueden ser analizados en las gráficas (Figs. 10 y 11): 


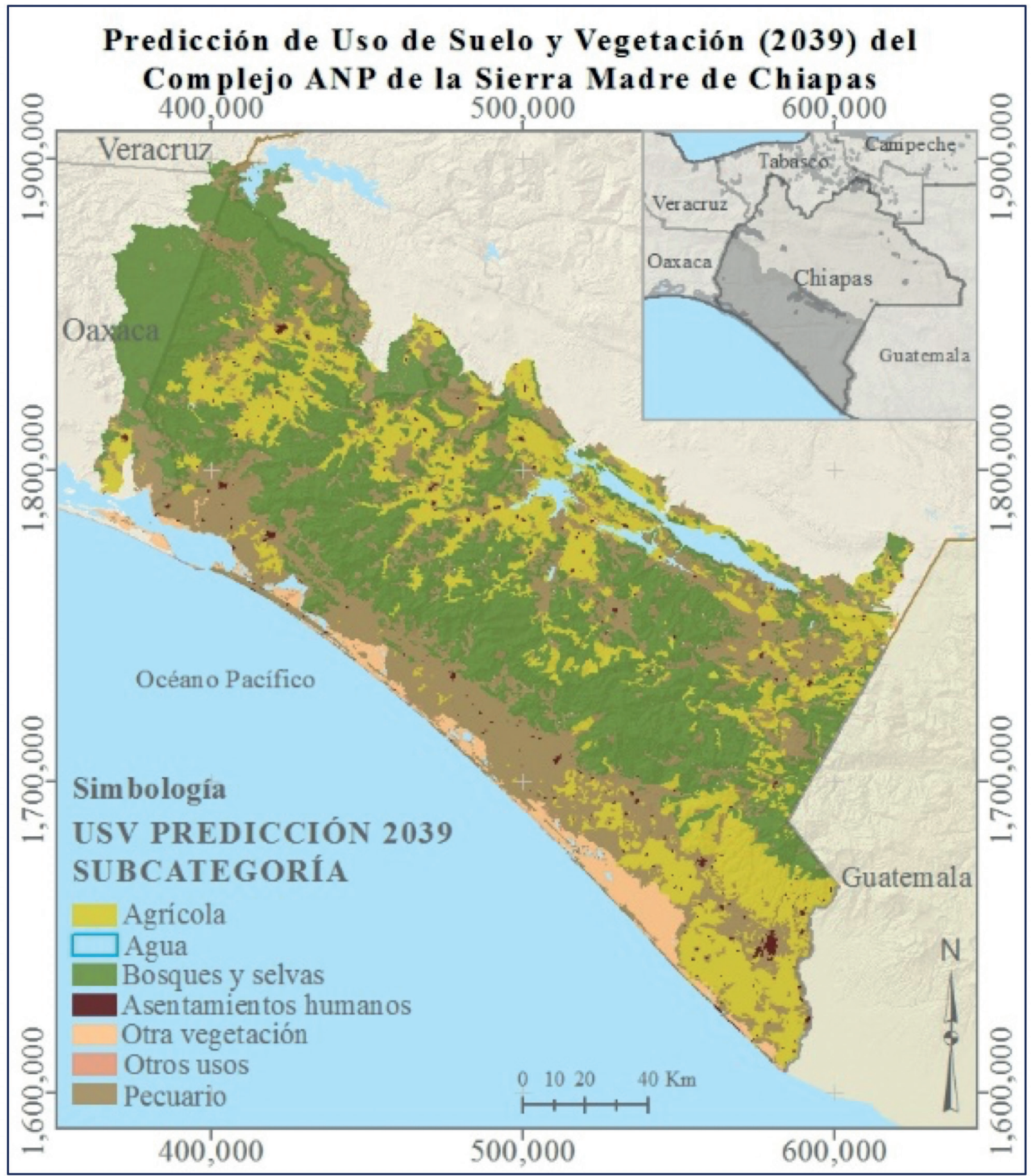

Figura 9. Escenario de USV para el año 2039 del Complejo de ANP de la Sierra Madre de Chiapas. Elaboración propia. 


\section{PRINCIPALES CAMBIOS EN EL USV DENTRO DE LAS ANP POR PORCENTAJE (\%)}

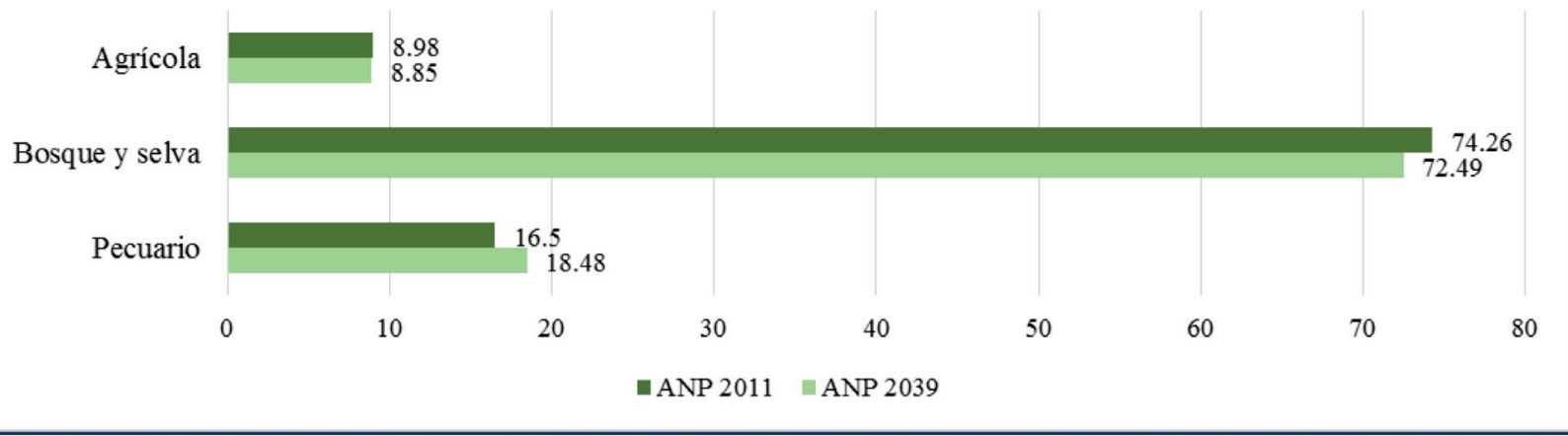

Figura 10. Cambios en el Uso de Suelo y Vegetación dentro de las ANP del año 2011 al 2039. Elaboración propia.

Los cambios de USV dentro de las ANP se centran en las categorías pecuario, agrícola y bosques y selvas, donde la categoría pecuario crece cerca del 1.9\%, mismo que se obtiene de las pérdidas de agrícola con $0.13 \%$ y bosques y selvas con $1.77 \%$ con un total de 700,000 ha. En toda el área de estudio la categoría pecuario es la que ganaría mayor territorio a diferencia de las demás categorías, como se mencionó con respecto de bosques y selvas, debido a que es una de las principales actividades económicas que utilizan mayor superficie para su desarrollo.

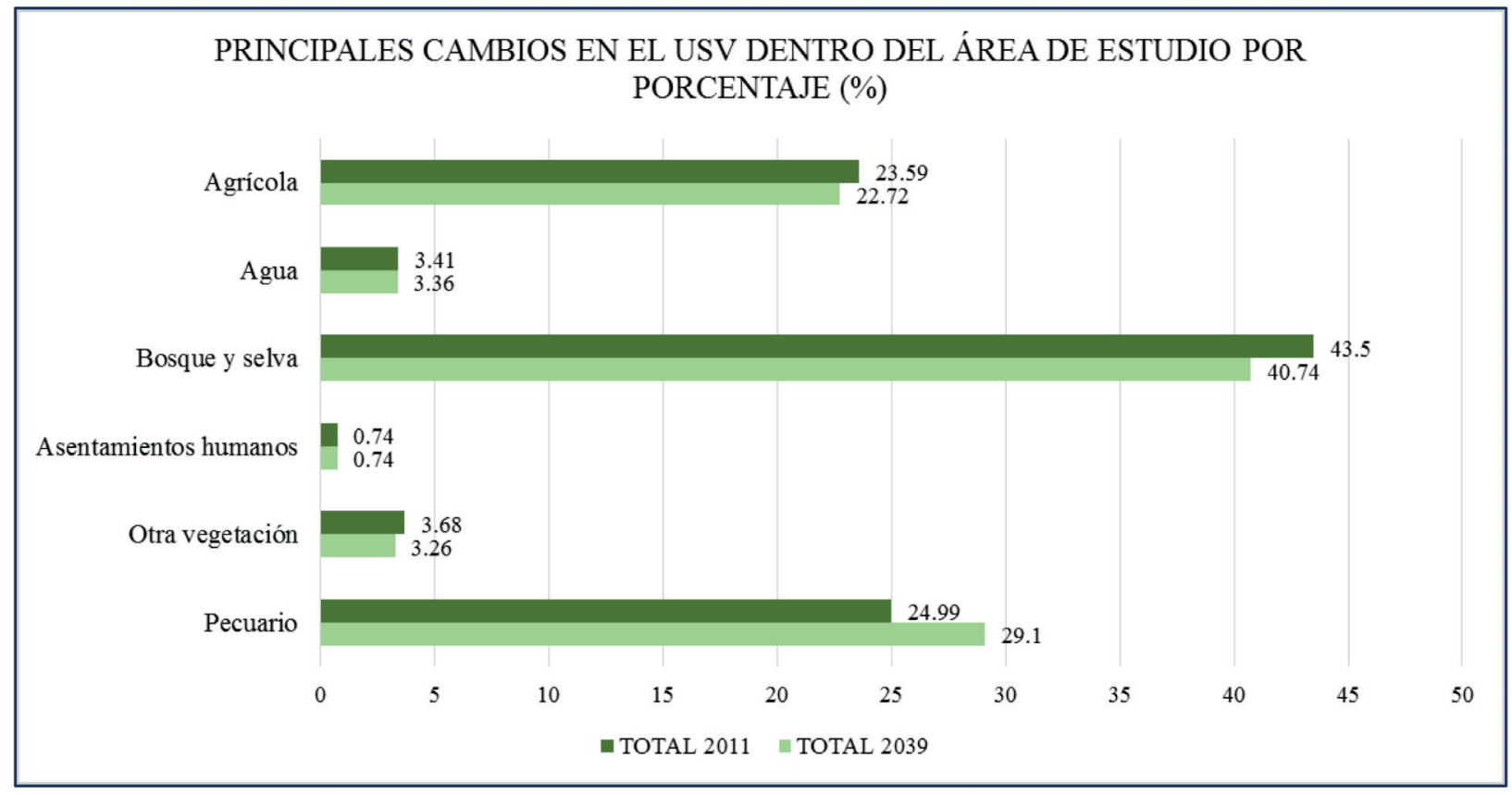

Figura 11. Cambios en el Uso de Suelo y Vegetación dentro del área de estudio del año 2011 al 2039. Elaboración propia. 
Comparando los resultados del cambio de USV entre los años 2011 y 2039 dentro del corredor, se observa el crecimiento de la categoría pecuario y en menor medida la de agricultura; sin embargo, pese a estas amenazas el $90 \%$ de los bosques y selvas dentro del corredor se preservarían de acuerdo con los resultados del escenario tendencial. Adicionalmente, para comprobar la utilidad del corredor se manejaron avistamientos del Tapir registrados por la CONABIO, reunidos mediante revisiones científicas, colectas en campo y fuentes bibliográficas, comprobando que el corredor propuesto es una zona útil para la conservación de esta especie.

Así los resultados obtenidos, bajo un enfoque de valoración de servicios ecosistémicos, permiten construir una propuesta para mantener la conectividad del tapir (Tapirus bairdii) en la región de la Sierra Madre de Chiapas y dirigir los esfuerzos de conservación a los sitios más vulnerables identificados en este trabajo. Si bien, nuestros resultados sugieren que la mayor parte del hábitat potencial para la especie está protegido y así podría seguir en un futuro cercano, se observan algunas áreas fuera de las ANP que podrían incorporarse dentro de una estrategia de conservación de la región. Finalmente, el corredor identificado en este trabajo contiene cantidades significativas de bosques y selvas que de acuerdo con el modelo tendencial van a permanecer en el futuro cercano, por lo que el corredor propuesto garantiza la conectividad para la población de tapires.

\section{Conclusiones}

Bajo el enfoque de la valoración de los servicios ecosistémicos y la obtención y procesamiento de información geoespacial dentro de un ambiente SIG se pudo obtener información espacialmente explícita para la planificación y toma de decisiones en relación con la conservación de los ecosistemas naturales, la diversidad biológica que contienen y los servicios ecosistémicos que nos brindan. Lo anterior, debido a que todas las herramientas empleadas en este trabajo están plenamente al análisis de los ecosistemas y pudieron relacionarse entre sí para obtener mejores resultados.

En este trabajo, el mapa de degradación juega un papel importante para entender la importancia del resguardo de las Áreas Naturales Protegidas, ya que son las zonas en donde se presenta mayor conservación de los usos de suelo y vegetación. Así mismo, parte del insumo principal para el diseño del corredor a partir de las zonas núcleo de La Sepultura y El Triunfo 
ya que presentan un porcentaje de calidad de hábitat alto, que aunque, se ve amenazado por el crecimiento de la población y las redes viales que están localizadas principalmente dentro de las zonas de amortiguamiento, este no tendría cambios significativos ni se vería fragmentado dentro del escenario proyectado, lo que demuestra su efectividad. Dado que una porción del corredor se encuentra fuera de las ANP esta parte carece de la protección jurisdiccional que brinda este instrumento, por lo que sería viable la ampliación y subzonificación de la reserva La Frailescana, con la finalidad que se le proporcione seguridad y mantenimiento al corredor y a los beneficios que esta estrategia de conservación conlleva.

\section{Agradecimientos}

Los autores agradecen al Fondo Sectorial de Investigación Ambiental SEMARNAT-CONACYT por el financiamiento del proyecto SEMARNAT-2015-1-263289. “Valoración económica de servicios ecosistémicos en el complejo de Áreas Naturales Protegidas de la Sierra Madre de Chiapas".

\section{Literatura citada}

Boll, J., van Buren, D., Campbell, C., Brooks, E., Chen, S., Stockle, C., McCool, D. 2004. Distributed modeling and economic analysis of erosion in GIS for watershed restoration. In: Paper Presented at the Integrated Decision-Making for Watershed Management Symposium, Maryland 7-9 January 2001. Consultado: 01-10-2016. En: http://www.bee.cornell.edu/ swlab/SoilWaterWeb/publications/BollIDMWM01.pdf

Comisión Nacional de Áreas Naturales Protegidas (CONANP). 2015. Valoración de Servicios Ecosistémicos: Un Enfoque para Fortalecer el Manejo de las Áreas Naturales Protegidas Federales de México. Comisión Nacional de Áreas Naturales Protegidas, Secretaría de Medio Ambiente y Recursos Naturales. México. 131 pp.

Comisión Nacional para el Conocimiento y Uso de la Biodiversidad (CONABIO). 2010. Portal de geoinformación, sistema nacional de información sobre biodiversidad, 'Tapirus bairdii (tapir Centroamericano). Distribución conocida, México. Consultado: 05-12-2016. En: http://www. conabio.gob.mx/informacion/gis/ 
Cortina-Villar, S., Plascencia-Vargas, H., Vaca, R., Schroth, G., Zepeda, Y., Soto-Pinto, L., \& NahedToral, J. 2012. Resolving the conflict between ecosystem protection and land use in protected areas of the Sierra Madre de Chiapas, Mexico. Environmental management, 49(3), 649-662.

Eade, J. D., \& Moran, D. (1996). Spatial economic valuation: benefits transfer using geographical information systems. Journal of Environmental management, 48(2), 97-110.

Eastman, R. J. 2006. IDRISI Andes Guide to GIS and Image Processing. Clark Labs, Clark University. Worcester, MA, US. 327 pp.

Foerster, C.R. 1998. Ecología de la danta centroamericana (Tapirus bairdii) en el Parque Nacional Corcovado, Costa Rica. Tesis de Maestría, Universidad Nacional, Heredia, Costa Rica.

Grêt-Regamey, A., P. Bebi, I. D. Bishop, y W. A. Schmid. 2008. Linking GIS-based models to value ecosystem services in an Alpine region. Journal of environmental management. 89(3), 197-208.

Instituto Nacional de Estadística y Geografía (INEGI). 1990. Censo General de Población y Vivienda 1990 Principales resultados por localidad (ITER). Consultado: 01-10-2016. En: http:/ /www. inegi.org.mx/sistemas/consulta_resultados/iter1990.aspx?c=2 7439\&s=est

Instituto Nacional de Estadística y Geografía (INEGI). 1997. Conjunto de datos vectoriales de la carta de Uso de Suelo y Vegetación. Escala 1:250 000. Serie II. Consultado: 03-05-2016. En: http://www.beta.inegi.org.mx/temas/mapas/usosuelo/

Instituto Nacional de Estadística y Geografía (INEGI). 2010. Censo de Población y Vivienda 2010 Principales resultados por localidad (ITER). Consultado: 12-11-2016. En: http:/ / www.inegi. org.mx/sistemas/consulta_resultados/iter2010.aspx?c=27329\&s=est

Instituto Nacional de Estadística y Geografía (INEGI). 2013a. Conjunto de datos vectoriales de la carta de Uso de Suelo y Vegetación. Escala 1:250 000. Serie V. Consultado: 03-05-2016. En: http://www.beta.inegi.org.mx/temas/mapas/usosuelo/

Instituto Nacional de Estadística y Geografía (INEGI). 2013b. Continúo de Elevaciones Mexicano 3.0 (CEM 3.0). Consultado: 14-11-2016. En: http://www.inegi.org.mx/geo/contenidos/ datosrelieve/continental/continuoelevacion es.aspx

Jenness, J., D. Majka y P. Beier. 2011. Corridor designer evaluation tools. Environmental Research, Development and Education for the New Economy (ERDENE). Northern Arizona University. Flagstaff, AZ. 103 pp.

Laterra, P., Nahuelhual, L., Parral, M., \& Carmona A. 2016. ECO-SER Protocolo colaborativo de 
evaluación y mapeo de servicios ecosistémicos y vulnerabilidad socio-ecológica para el ordenamiento territorial. Consultado: 25-04-2016. En: http:/ / www.eco-ser.com.ar/

March, I.J. y E.J. Naranjo. 2005. Tapir (Tapirus bairdii). pp. 496-497. En: Ceballos, G y G. Oliva (Eds.). Los mamíferos silvestres de México. Comisión Nacional para el Conocimiento y Uso de la Biodiversidad, y Fondo de Cultura Económica, Ciudad de México, México. 986 pp.

Martín-López, B., M. García-Llorente, I. Palomo y C. Montes. 2011. The conservation against development paradigm in protected areas: Valuation of ecosystem services in the Donana socialecological system (southwestern Spain). Ecological Economics. 70 (8): 1481-1491.

Mas, J. F., M. Kolb, M. Paegelow, M. T. C. Olmedo y T. Houet. 2014. Inductive pattern-based land use/cover change models: A comparison of four software packages. Environmental Modelling \& Software. 51: 94-111.

Mendoza-Ramírez, E. y J. P. Carbajal Borges. 2011. Avances y perspectivas para la conservación del tapir centroamericano en México. CONABIO. Biodiversitas. 99: 12-16.

Naranjo, E.J. y E. Cruz. 1998. Ecología del tapir (Tapirus bairdii) en la reserva de la biósfera La Sepultura, Chiapas, México. Acta Zoológica Mexicana (nueva serie). 73: 111-125.

Nemec, K. T. y C. Raudsepp-Hearne. 2013. The use of geographic information systems to map and assess ecosystem services. Biodiversity and conservation. 22 (1): 1-15.

Sharp, R., H. T. Tallis, T. Ricketts, A. D. Guerry, S. A. Wood, R. Chaplin-Kramer, E. Nelson, D. Ennaanay, S. Wolny, N. Olwero, K. Vigerstol, D. Pennington, G. Mendoza, J. Aukema, J. Foster, J. Forrest, D. Cameron, K. Arkema, E. Lonsdorf, C. Kennedy, G. Verutes, C. K. Kim, G. Guannel, M. Papenfus, J. Toft, M. Marsik, J. Bernhardt, R. Griffin, K. Glowinski, N. Chaumont, A. Perelman, M. Lacayo, L. Mandle, P. Hamel, A. L. Vogl, L. Rogers, W. Bierbower, D. Denu y J. Douglass. 2016. InVEST + VERSION+ User's Guide. The Natural Capital Project, Stanford University, University of Minnesota, The Nature Conservancy, and World Wildlife Fund. 339 pp.

Sarukhán, J., J. Carabias, P. Koleff y T. Urquiza-Haas. 2012. Capital natural de México: Acciones estratégicas para su valoración, preservación y recuperación. Comisión Nacional para el Conocimiento y Uso de la Biodiversidad (CONABIO). Ciudad de México, México. 91 pp. Tallis, H.T., T. Ricketts, A.D. Guerry, S.A. Wood, R. Sharp, E. Nelson, D. Ennaanay, S. Wolny, N. Olwero, K. Vigerstol, D. Pennington, G. Mendoza, J. Aukema, J. Foster, J. Forrest, D. Cameron, 
K. Arkema, E. Lonsdorf, C. Kennedy, G. Verutes, C.K. Kim, G. Guannel, M. Papenfus, J. Toft, M. Marsik, J. Bernhardt, y R. Griffin. 2013. InVEST 2.5.3 User's Guide. The Natural Capital Project, Stanford. Consultado: 22-02-2015. En: http://ncp-dev.stanford.edu/ dataportal/ invest-releases/documentation/current_release/

Troy, A., y M. A. Wilson. 2006. Mapping ecosystem services: practical challenges and opportunities in linking GIS and value transfer. Ecological economics. 60(2), 435-449.

Vazquéz-Bautista, D. 2002. Mastofauna de la zona de protección forestal La Frailescana, Chiapas, México. Tesis de Licenciatura. Universidad de Ciencias y Artes de Chiapas.

Villa, F., et al., (2007). Villa, F., Bagstad, K., Voigt, B., Balbi, S., Athanasiadis, I., Willcock, S., Pascual, M., Martínez-López, J., Azkarate, A., y Scott, L. 2007. Artificial Intelligence for Ecosystem Services (ARIES). Consultado: 28-04-2016. En: http:/ / aries.integratedmodelling.org/

Cita:

Medina-Fernández S.L., C. Ordoñez-Ramos y J. M. Núñez*. 2018. Modelado de Hábitat, Corredores y Escenarios para la Valoración de Servicios Ecosistémicos en Áreas Naturales Protegidas de la Sierra Madre de Chiapas. 2018. Vol. 4 (1): 25-47. https:/ / doi.org/10.18242/ anpscripta.2018.04.04.01.0002

Sometido: 16 de Octubre de 2017

Revisado: 17 de Noviembre de 2017

Aceptado: 18 de Enero de 2018

Editor asociado: Dr. Miguel Ángel Pinkus Rendón

Idioma Inglés Abstract: Ms.C. Diana Dorantes

Diseño gráfico editorial: Lic. Gerardo Hernández 